2. Ч $е$ в в $\times$ К.Ф. Линейная гесрия оболочек.Ч.І, Л., ЛТИ, I962.

АСИМТОТИЧЕККО ИССЛЕДОВННИЕ ДЕФОРМАLИЙ

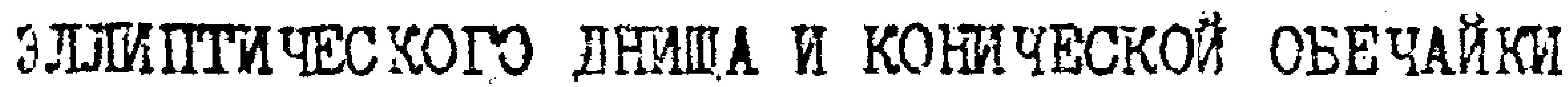

\title{
E.H. GKOBREB
}

Рассматривается деформапия әллитти ческого дпка * кови-

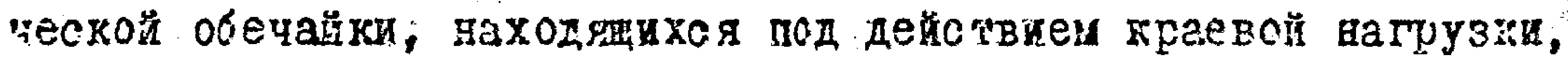
rсторая изменяется по захояу cosky илt sink:.

Для случая произвольного еагрухения ка краю оболочки

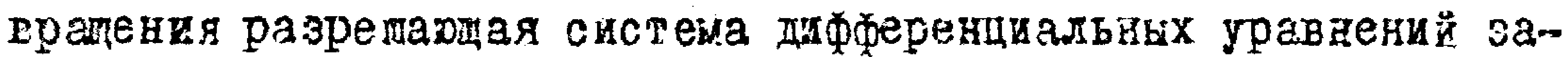

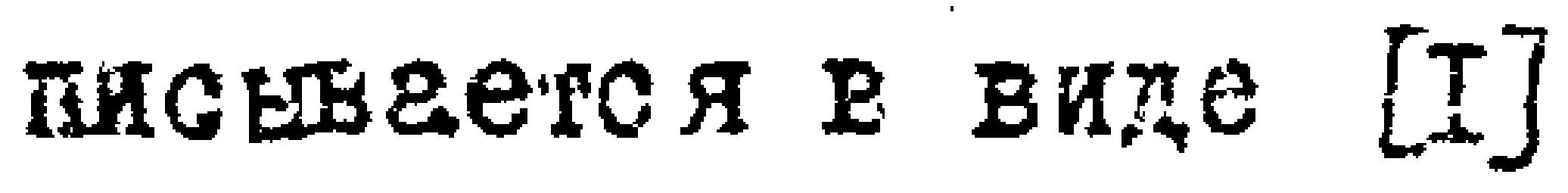

$$
\begin{aligned}
& G_{k}\left\{\tilde{U}_{, k}\right\}+k^{2}\left[1-i c\left(\frac{1}{R_{1}}-\frac{1}{R_{2}}\right) \frac{1}{\sin ^{2} \theta}\right] \tilde{T}_{, k}=0 ; \\
& G_{k}\left\{\tilde{W}_{, k}\right\}-\frac{k^{2}}{\sin ^{2} \theta}\left(\frac{1}{R_{1}}-\frac{1}{R_{2}}\right) \tilde{W}_{k}=0,
\end{aligned}
$$

rae

$$
\begin{aligned}
& G_{k}=\frac{1}{R_{1} R_{2}} \frac{d}{d \theta}\left(\frac{R_{2}^{2} \sin \theta}{R_{1}} \frac{d}{d \theta}\right)-\frac{k^{2}}{R_{2} \sin ^{2} \theta} ; \\
& \tilde{W}_{k}=\tilde{U}_{i k}+i c k^{2} \tilde{T}_{, k} ; \quad c=h / \sqrt{12\left(i-\nu^{2}\right)} ;
\end{aligned}
$$

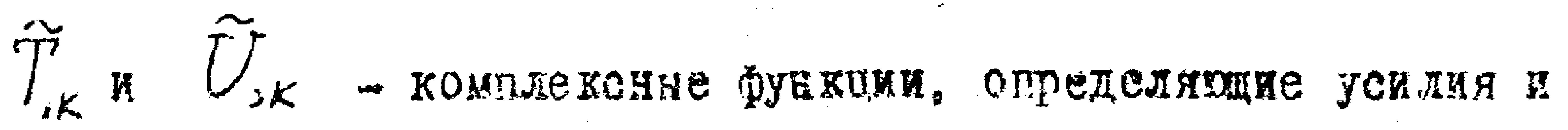
моменти; $R_{1}$ и $R_{2}$ - радиусы хривизны срединной поверх-

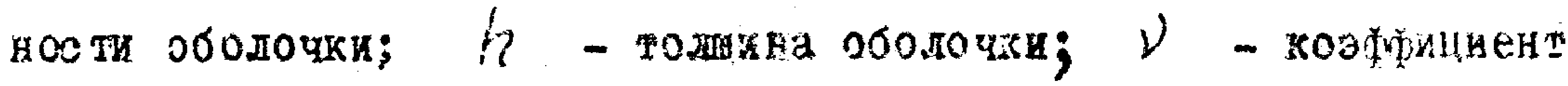
Пауссока; $\theta$ - угол, осразуемнй вориальо х сеединай по-

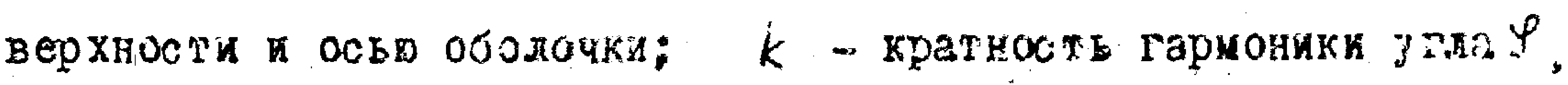

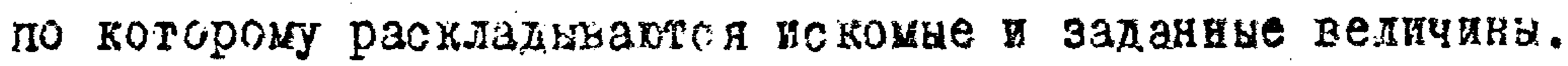

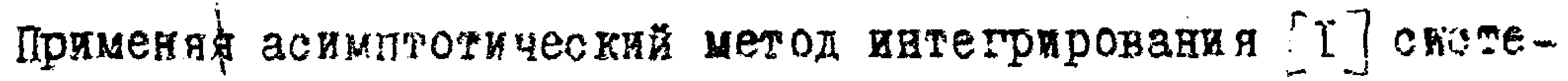


ив (I), пюлучим решендя для ободочек вращеняя в виде наломения дБух типов решенй.

I. Решения для хонической оболочки запиттся в виде

a)

$$
\begin{array}{ll}
\widetilde{U_{, k}}=A\left(1-\frac{i c k^{2}}{\operatorname{scos} \alpha \sin \alpha}\right) ; & \sigma) \widetilde{U_{, k}}=-i c k^{2} \widetilde{i_{1}}, \\
\widetilde{T_{, k}}=\tilde{A}(s \cos \alpha \sin \alpha)^{-1} ; & \tilde{T_{, k}}=\tilde{B} e^{\beta_{s},}
\end{array}
$$

Где

$$
\begin{aligned}
& \beta_{0}=-(k / \operatorname{ctg} \alpha)^{1 / 2}\left(b_{1}^{(c)}-i b_{i}^{(c)}\right)\left(\sqrt{s}-\sqrt{s_{0}}\right) ; \\
& b_{1}^{(c)}=1+\frac{c k^{2}}{s_{0} \cos \alpha \sin \alpha} ; \quad g_{2}^{(c)}=1-\frac{c k^{2}}{s_{0} \cos \alpha \sin \alpha} ;
\end{aligned}
$$

$\alpha=\frac{\pi}{2}-\theta$ - угол конусвоста, $s$ - расстояне вдоль обран зуюией конуса, $S_{0}$ - значение координату, отвечариее верхнену крап усеченного жонуса, $\tilde{A}=A_{1}+i A_{2}$;

$$
\tilde{B}=B_{1}+i B_{2}^{\prime}
$$

- комплексные погтоянин.

Demeri: (2) согласно формул [2], после соответствудй операий разделения действительных и миимх частей, поэволяот найти перенещения в виде

$$
T_{r_{c}}^{(c)}=A_{1} \psi_{n}^{(c)}+A_{2} \psi_{n}^{(c)}+B_{1} \Phi^{(c)}+B_{2} \Phi^{(c)}
$$

Siness

$$
\begin{aligned}
& T_{n}^{(c)}=\left\{\begin{array}{ccc}
U_{, k} ; & V_{, k} ; & W_{, k} \\
1 & 2 & 3
\end{array}\right\} ; \\
& \Psi_{1}^{\prime(c)}=-\frac{(1+\nu) c}{E h} \frac{1}{s \cos ^{2} \alpha} ; \quad \Psi_{2}^{\prime(c)}=\frac{k}{\sin x} \Psi_{1}^{(c)}
\end{aligned}
$$

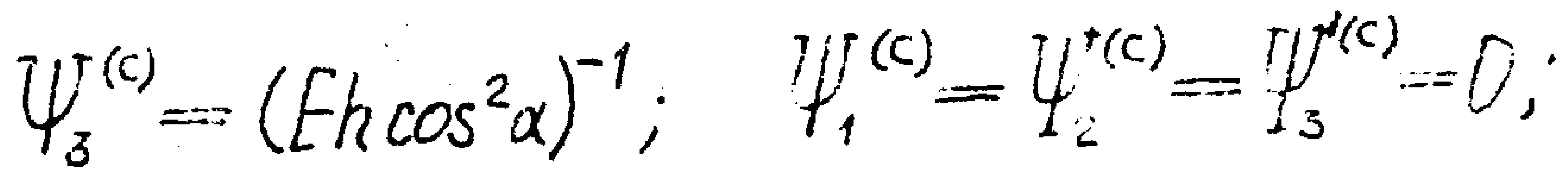

$$
\begin{aligned}
& \Phi_{1}^{(c)}=\frac{(1+\nu) c}{E ! h} s \operatorname{tg} \alpha e^{R\left(\beta_{s}\right.} \Psi_{s} ; \quad \Phi_{1}^{\prime(c)}=\frac{\varphi_{s}^{\prime}}{\varphi_{s}} \Psi_{1}^{(c)} ;
\end{aligned}
$$

$T 58$ 


$$
\begin{aligned}
& \Phi_{2}^{(c)}=-\frac{(1+\nu) c}{E h} \frac{k}{\cos \alpha} e^{R e \beta_{s}} \sin \operatorname{Im} \beta_{s} ; \Phi_{2}^{\prime(c)}=-\operatorname{ctg} \operatorname{Im} \beta_{s} \Phi_{2}^{(c)}, \\
& \Phi_{3}^{(c)}=\frac{s \operatorname{tg} \alpha}{E h} e^{R e \beta_{s}} \cos \operatorname{Im} \beta_{s} ; \quad \Phi_{3}^{\prime(c)}=-\operatorname{tg} \operatorname{Im} \beta_{s} \Phi_{3}^{(c)},
\end{aligned}
$$

rae

$$
\begin{aligned}
& \varphi_{s}=\cos \operatorname{Im} \beta_{s} \cdot \operatorname{Im} \beta_{s}^{\prime}+\sin \operatorname{Im} \beta_{s} \cdot \operatorname{Re} \beta_{s}^{\prime} ; \quad \operatorname{Re} \beta_{s}^{\prime}=-(2 \operatorname{cstg} \alpha)^{-1 / 2} g_{1}^{(c)} ;
\end{aligned}
$$

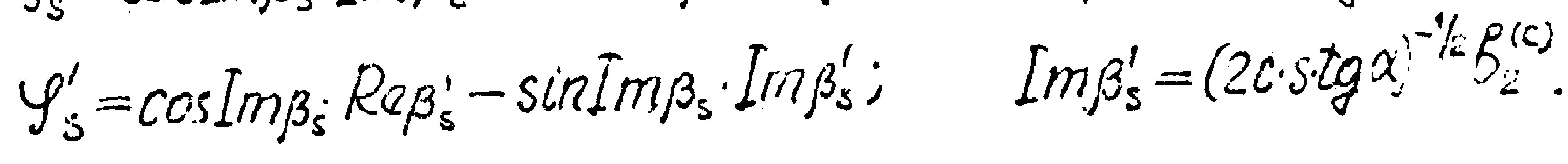

I. Аспмптоличес кие решения для эллития пескон оболочки представим как

a)

$$
\begin{aligned}
& \tilde{U}_{, k}=\tilde{A}\left[1-i c k^{2} \tau(\theta)\right] ; \\
& \text { б) } \tilde{U}_{, k}=-i c k^{2} \widetilde{T}_{, k} \text {; } \\
& \widetilde{T}_{, k}=\tilde{A} \tilde{i}(\theta) e^{-\beta^{*}} ; \\
& \tilde{T}_{, k}=\tilde{B} e^{-\beta_{0}} \text {, }
\end{aligned}
$$

где

$$
\begin{aligned}
& \beta^{*}=k \ln \left\{\frac{\sin \theta}{\sin \theta_{0}} \frac{\left(1+\gamma \sin ^{2} \theta_{0}\right)^{1 / 2}+\cos \theta_{0}}{\left(1+\gamma \sin ^{2} \theta\right)^{1 / 2}+\cos \theta}\right\} ; \\
& \beta_{\theta}=\left(\frac{r_{0}}{2 c}\right)^{1 / 2} \frac{(1+\gamma)^{1 / c}}{\left(1+\gamma \sin ^{2} \theta_{0}\right)^{s / 4}}\left(t_{1}^{(e)}-i b_{2}^{(\theta)}\right)\left(\theta_{0}-\theta\right) .
\end{aligned}
$$

DAec

$$
\begin{aligned}
& b_{1}^{(e)}=1+\frac{k^{2}}{2 \sin ^{2} \theta_{0}} \frac{c}{r_{0}}\left(\frac{1+\gamma \sin ^{2} \theta_{0}}{1+\gamma}\right)^{1 / 2}\left(1-\gamma \sin ^{2} \theta_{0}\right) ; \\
& b_{2}^{(e)}=1-\frac{k^{2}}{2 \sin ^{2} \theta_{c}} \frac{c}{r_{0}}\left(\frac{1+\gamma \sin ^{2} \theta_{0}}{1+\gamma}\right)^{1 / 2}\left(1-\gamma \sin ^{2} \theta_{0}\right) ; \\
& \tau(\theta)=-\gamma / R_{2} ; \quad \gamma=(a / b)^{2}-1 ;
\end{aligned}
$$

I5: 
$a, b$ - большая и малая подуоси әлгипа.

Иопользуя соотнопения общей теори тонкостенных о0оло-

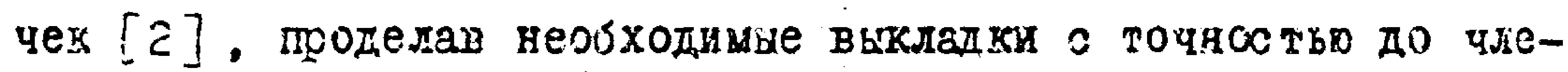
нов порядка $\left(i / r_{0}\right)^{1 / 2}$ и отденяя действительдуп и милмур части, получим әьралевия перемешений в виде

$$
T_{n}^{(\varepsilon)}=A_{1} \psi_{n}^{(\alpha)}+A_{2} \psi_{n}^{\prime(\alpha)}+B_{1} \Phi_{n}^{(\varepsilon)}+B_{2} \Phi_{n}^{(e)}
$$

Здес b

$$
T_{n}^{(e)}=\left\{\begin{array}{ccc}
u, k ; & V, k ; & W, k \\
1 & 2 & 3
\end{array}\right\} ;
$$

$\left.\Psi_{1}^{(\alpha)}=\frac{(1+\nu) C}{E h} \tau_{1}(\theta) e^{-\beta^{*}} ; \quad T_{1}^{\prime}\right)^{\prime(Q)}=\mp \frac{(1+\nu) c}{E h} \cdot \frac{k}{\sin \theta} \tau(\theta) e^{-\beta^{*}} ;$

$\psi_{3}^{\left(\mathcal{H}^{(}\right)}=\left(R_{1} / E h\right) \tau(\theta) e^{-\beta^{*}}$

$$
\psi_{1}^{(e)}=\Psi_{2}^{(e)}=\psi_{3}^{\prime(e)}=0 \text {; }
$$

$\Phi_{1}^{(\varepsilon)}=-\frac{(1+\nu) c}{E h} \varphi_{\theta}^{\prime} \cdot e^{-\operatorname{Re} \beta_{\theta}} ; \quad \Phi_{1}^{\prime(\ell)}=-\frac{(1+\nu) c}{E h} \varphi_{\theta} e^{-R e \beta_{\theta}} ;$

$\Phi_{2}^{(e)}= \pm \frac{(1+v) c}{E h} \frac{k}{\sin \theta} \sin \operatorname{Im} \beta_{\theta} e^{-R\left(\varepsilon \beta_{\theta}\right.}$;

$\Phi_{2}^{\prime(e)}=\mp \frac{(1+\nu) c}{E h}-\frac{k}{\sin \theta} \cos \operatorname{Im} \beta_{\theta} e^{-\operatorname{Ra} \beta_{\theta}}$;

$\Phi_{3}^{(e)}=\frac{R_{1}}{E h} \cos \operatorname{Im} \beta_{\theta} e^{-R_{2} \beta_{\theta}} ; \Phi_{3}^{\prime(e)}=\frac{R_{1}}{E h} \sin \operatorname{Im} \beta_{\theta} e^{-R e \beta_{2}}$,

где $\varphi_{\theta}=\cos \operatorname{Im} \beta_{\theta} \cdot \operatorname{Re} \beta_{\theta}^{\prime}+\sin \operatorname{Im} \beta_{\theta} \cdot \operatorname{Im} \beta_{\theta}^{\prime} ;$

$$
\varphi_{\theta}^{\prime}=\cos \operatorname{Im} \beta_{\theta} \operatorname{Im} \beta_{\theta}^{\prime}-\sin \operatorname{Im} \beta_{\theta} \cdot \operatorname{Re} \beta_{\theta}^{\prime} ;
$$

$R \alpha \beta_{\theta}^{\prime}=-\sqrt{\frac{r_{0}}{2 C}} \frac{(1+\gamma)^{1 / 2}}{\left(1+\gamma \sin ^{2} \theta_{0}\right)^{5 / 4}} b_{1}^{(\alpha)} ; \operatorname{Im} \beta_{\theta}^{\prime}=\sqrt{\frac{r_{0}}{2 C}} \frac{(1+\gamma)^{1 / 2}}{\left(1+\gamma \sin ^{2} \theta_{0}\right)^{5 / 4}} b_{2}^{(\alpha)} ;$

$$
\tilde{u}_{1}(\theta)=\gamma k / k_{0} \sin \theta \text {. }
$$


Формулы (3) и (4), с точностьр до членов порядта $\left(c / r_{0}\right)^{1 / 2}$ совпадаол с аналогичными выражениями для $k=1$ из работы $[E]$.

$$
\text { Ли т } е \text { р ат у р a }
$$

I. Н О в о пи ло о в В.В. Теория тонких оболочек. Л., Судпромги 3, I96?.

2. Ч е $\mathrm{p}$ и в $\mathrm{x}$ К. Ф. Линейная теория оболочек. Ч.І. Л., $\pi \Gamma y$, I952. 